\title{
Some Conceptual Difficulties Regarding 'Net' Multipliers
}

\author{
Ferran Sancho \\ Department of Economics \\ Universitat Autònoma de Barcelona \\ 01893-Bellaterra, Catalonia, Spain \\ ferran.sancho@uab.cat
}

\begin{abstract}
Multipliers are routinely used for impact evaluation of private projects and public policies at the national and subnational levels. Oosterhaven \& Stelder (2002) correctly pointed out the misuse of standard 'gross' multipliers and proposed the concept of 'net' multiplier as a solution to this bad practice. We prove their proposal is not well founded. We do so by showing that supporting theorems are faulty in enunciation and demonstration. The proofs are flawed due to an analytical error but the theorems themselves cannot be salvaged as generic, non-curiosum counterexamples demonstrate. We also provide a general analytical framework for multipliers and, using it, we show that standard 'gross' multipliers are all that is needed within the interindustry model since they follow the causal logic of the economic model, are well defined and independent of exogenous shocks, and are interpretable as predictors for change.
\end{abstract}

Keywords: gross multipliers, net multipliers, interindustry accounting, interindustry models.

JEL: C67, D57, 022, R15

Contact information: Phone: +34935811757, Fax:+34935812012, Email: ferran.sancho@uab.cat. 


\section{INTRODUCTION}

Not long ago a lively and sometimes quite heated debate ensued in the literature resulting from the publication of the Oosterhaven \& Stelder (2002) article on 'net' multipliers (OS henceforth). 'Net' multipliers were introduced as possible substitutes and correctors for the standard and common 'gross' multipliers, which were reportedly as being habitually misused in empirical applications of impact evaluations. Point in case is the OS description of the incorrect evaluation of the impact of the transport system in the Netherlands, with 'gross' multipliers and 'gross' output being used simultaneously, hence upward biasing its impact by double counting. We agree with OS that these professional malpractices should be avoided but disagree on their proposal on theoretical grounds. In point of fact, OS proposal is conceptually faulty and the supporting theorems are incorrect, as simple, non-curiosum counterexamples testify.

Chronologically the essence of the scientific debate can be followed in De Mesnard (2002, 2007a, 2007b), Dietzenbacher (2005), and Oosterhaven (2004, 2007a, 2007b). We share De Mesnard's concern that OS 'net' multipliers are empirically unstable since they depend upon the magnitude of the exogenous shock; hence they are hardly usable in rigorous quantitative analysis. Due to their instability De Mesnard (2002, 2007a) concludes that they can only be used for the evaluation of small changes since then induced errors will also be small. Dietzenbacher (2005), in turn, tries to rationalise the discussion suggesting an interesting reinterpretation of the OS proposal as a set of parameters for the identification of strategic sectors. He also asserts that OS 'net' multipliers are as plausible as any other multipliers, conditional to the question they want to address. We disagree with Dietzenbacher's view of the problem regarding their reasonability. To give support to our alternative view, we will delve deeper into the conceptual root of the OS definitions. We shall argue that the definitions are ill founded for the claimed purpose and, in fact, they hardly belong to the category of multipliers since they have no natural interpretation in term of cause-effect, neither in economics nor in mathematics, and they do not capture economy-wide interaction effects. The implication is that OS 'net' multipliers cannot-should not - be used as predictors. Additionally, the purported supporting theorems of OS will be proved to be erroneous in enunciation and demonstration. Since 'net' multipliers are being used in empirical analysis (e.g. Márquez, 2011) and there are even attempts to extend them (e.g. Temurshoev \& Oosterhaven, 2010) we believe it is of paramount relevance to discuss and explicate their inner conceptual difficulties. 
The paper follows this organisation. Section 2 and 3 are the core sections. In Section 2 we elaborate some of the needed technical details and facts on standard multiplier analysis and, in their light, we recap OS proposal. Section 3 presents and discusses the main analytical results and economic counterarguments to OS. Section 4 briefly examines possible alternatives, if any. Section 5 concludes.

\section{DEFINITIONS AND BASIC FACTS ON MULTIPLIERS.}

It is fair to say that input-output economics can be credited with the introduction and extensive use of multiplier effects at the sectoral level. Conceptually, however, they very much belong to the same Keynesian tradition as the well-known multiplier of macroeconomics. The basic underlying idea is that, because of economy wide interactions, exogenous injections multiply themselves over and above their initial value once all equilibrium adjustments are internalised. Hence the 'multiplier' tag.

An input-output economy can be described by a tern $\left(\mathbf{Z}, \mathbf{v}^{\prime}, \mathbf{f}\right)$ where $\mathbf{Z}$ is an $n \times n$ matrix of intermediate bilateral exchanges, $\mathbf{v}^{\prime}$ a row $n$-vector of sectoral value-added and $\mathbf{f}$ a column $n$ vector of sectoral final demand. The balance identities guarantee that in any equilibrium:

$$
\sum_{j=1}^{n} z_{i j}+f_{i}=\sum_{j=1}^{n} z_{j i}+v_{i}=x_{i} \quad(i=1,2, \ldots, n)
$$

with the column $n$-vector $\mathbf{x}=\left(x_{1}, x_{2}, \ldots, x_{n}\right)$ indicating total demand (left expression) and total supply (middle one). All magnitudes are expressed in currency units. We can visualize these value magnitudes as though they were also physical magnitudes simply by using the standard normalisation, i.e. redefine all actual physical units (usually unobservable) so that each new unit has a worth of one currency unit. This has the definite advantage that the implicitly redefined physical units will all have unitary prices in the initial equilibrium. Balance data can now be unambiguously transformed into a model by way of defining and introducing a non-negative $n \times n$ matrix A of technical coefficients by:

$$
a_{i j}=\frac{z_{i j}}{x_{j}} \quad(i, j=1,2, \ldots, n)
$$


Expression (2) assumes fixed production coefficients and constant-returns-to-scale (CRS). From (2) we can rewrite the first part of (1) as:

$$
\sum_{j=1}^{n} z_{i j}+f_{i}=\sum_{j=1}^{n} a_{i j} \cdot x_{i}+f_{i}=x_{i} \quad(i=1,2, \ldots, n)
$$

which in matrix terms becomes:

$$
\mathbf{A} \cdot \mathbf{x}+\mathbf{f}=\mathbf{x}
$$

If matrix $\mathbf{A}$ is productive (satisfies the Hawkins-Simon condition or the equivalent less than unitary eigenvalue property; Nikaido, 1972, chapter 3) then (4) can be non-negatively solved for any non-negative vector $\mathbf{f}$ as:

$$
\mathbf{x}=(\mathbf{I}-\mathbf{A})^{-1} \cdot \mathbf{f}=\mathbf{L} \cdot \mathbf{f}
$$

with $\mathbf{L}$ being known as the Leontief inverse. Equation (5) is the mathematical representation of the classical interindustry quantity model of Leontief (1936). It is a system of $n$ linearly independent equations with $n$ unknowns; hence its non-negative solution is also unique.

Total income $v$ in this economy is given by aggregate value-added whereas total expenditure $f$ is given by aggregate final demand. From the national accounts identities (or from aggregating (1)) we know the following equality between income and net output will also hold true in any equilibrium:

$$
v=\sum_{j=1}^{n} v_{j}=\mathbf{v}^{\prime} \cdot \mathbf{e}=\mathbf{e}^{\prime} \cdot \mathbf{f}=\sum_{i=1}^{n} f_{i}=f
$$

In (6) the $n$-vector $\mathbf{e}\left(\mathbf{e}^{\prime}\right)$ represents the unit column (row) vector. Assuming that valueadded generation follows the same technical characteristics of fixed coefficients and CRS, we can introduce value-added technical coefficients $v_{c j}=v_{j} / x_{j}$, which using (5) allows us to write expression (6) as:

$$
v=\sum_{j=1}^{n} v_{j}=\sum_{j=1}^{n} \frac{v_{j}}{x_{j}} \cdot x_{j}=\sum_{j=1}^{n} v_{c j} \cdot x_{j}=\mathbf{v}_{c}^{\prime} \cdot \mathbf{x}=\mathbf{v}_{\mathbf{c}}^{\prime} \cdot \mathbf{L} \cdot \mathbf{f}
$$


where $\mathbf{v}_{\mathbf{c}}^{\prime}$ is the row $n$-vector of value-added technical coefficients. We now turn into introducing the standard or regular ('gross' in OS terminology) output and value-added multipliers ${ }^{1}$. Notice first that in the basic economic model of expression (5) causality runs from the vector of final demand $\mathbf{f}$ to the vector of total output $\mathbf{x}$. In the language of calculus, $\mathbf{f}$ would be termed as the independent variable and $\mathbf{x}$ as the dependent one. The mathematical equation governing the economic model in (5) is nothing but the linear mapping $\mathbf{L}: \mathrm{R}^{n} \rightarrow \mathrm{R}^{n}$ with $\mathbf{x}=\mathbf{L}(\mathbf{f})=\mathbf{L} \cdot \mathbf{f}$. Aggregate total output $x$ can then be obtained ${ }^{2}$ as $x=\mathbf{e}^{\prime} \cdot \mathbf{x}=\mathbf{e}^{\prime} \cdot \mathbf{L} \cdot \mathbf{f}$. The multiplier concept can therefore be seen as simply being calculus derivatives obtained from the mathematical representation of the basic economic model, or its variants.

Definition 1: Output multipliers. They will be denoted by an $n$-vector $\mathbf{m}_{x}^{\prime}$ and be defined as the derivative of total aggregate output $x$ with respect to final demand $\mathbf{f}$ :

$$
\mathbf{m}_{x}^{\prime}=\frac{d x}{d \mathbf{f}}=\frac{d\left(\mathbf{e}^{\prime} \cdot \mathbf{L} \cdot \mathbf{f}\right)}{d \mathbf{f}}=\mathbf{e}^{\prime} \cdot \mathbf{L}
$$

In words, each vector component $m_{x, j}$ corresponds to the sum of the respective column in the Leontief inverse matrix $\mathbf{L}$ :

$$
m_{x, j}=\sum_{i=1}^{n} \ell_{i j}
$$

so that for given $j$ the sum of all Leontief coefficients in column $j$ of $\mathbf{L}$ gives the aggregate effect on all sectors of a unitary exogenous increase in final demand for $j$. The numerical value $m_{x}^{\prime}$ indicates how the exogenous unitary injection in $j$ gets transmitted over the whole economic system, adding up all sectoral effects. From expression (5) it is also easy to see the calculus interpretation of multipliers:

$$
\frac{\partial x_{i}}{\partial f_{j}}=\ell_{i j}
$$

\footnotetext{
${ }^{1}$ We will focus on so-called type I multipliers. Similar considerations would apply to type II multipliers but we omit any references to them for the sake of brevity.

${ }^{2}$ Since prices are independent of quantities in the model, aggregation entails using reference prices which are kept hidden because they play no explicit role and are set to unity using the standard normalization.
} 
All the information required to calculate the output multipliers is contained in matrix $\mathbf{L}$ and this explain why this matrix is commonly referred to as the output multiplier matrix.

Definition 2: Value-added multipliers. They will be denoted by an $n$-vector $\mathbf{m}_{v}^{\prime}$ and be defined as the derivative of total value added $v$ with respect to final demand f. Using expression (7) we would obtain:

$$
\mathbf{m}_{v}^{\prime}=\frac{d v}{d \mathbf{f}}=\frac{d\left(\mathbf{v}_{\mathbf{c}}^{\prime} \cdot \mathbf{L} \cdot \mathbf{f}\right)}{d \mathbf{f}}=\mathbf{v}_{\mathbf{c}}^{\prime} \cdot \mathbf{L}
$$

Each $j$ component of this multiplier vector measures the overall effect on value-added that would follow from a unitary increase in final demand for $j$ and can therefore be visualised as:

$$
m_{v, j}=\frac{\partial v}{\partial f_{j}}=\sum_{i=1}^{n} v_{c i} \cdot \ell_{i j}
$$

By using (12) we can evaluate the economy-wide effect on total value-added resulting from a unitary increase in final demand for good $j$.

Observe the nice formal similarity between output and value-added multipliers. For valueadded, they are computed as the product of a vector of ratios (of value added to total output, i.e. $\mathbf{v}_{\mathbf{c}}^{\prime}$ ) and the matrix $\mathbf{L}$ capturing direct and indirect production interdependencies. The same applies to output multipliers. In this case the vector of ratios is the unit vector $\mathbf{e}^{\prime}$ (trivially, of total output to total output). This allows for an easy and straightforward generalisation to other multipliers. Should we be interested in employment multipliers, we would then need the vector of ratios of employment $\mathbf{w}$ to total output $\mathbf{x}$; say such vector of employment coefficients is $\mathbf{w}_{\mathbf{c}}^{\prime}$, then employment multipliers would be given by the vector $\mathbf{m}_{w}^{\prime}=\mathbf{w}_{\mathbf{c}}^{\prime} \cdot \mathbf{L}$. Or consider carbon dioxide emissions $\mathbf{d}$, with sectoral ratios of $\mathrm{CO}_{2}$ emissions to total output being picked up by a vector of emission coefficients $\mathbf{d}_{\mathbf{c}}^{\prime}$; then carbon dioxide emission multipliers would be computed using the expression $\mathbf{m}_{d}^{\prime}=\mathbf{d}_{\mathbf{c}}^{\prime} \cdot \mathbf{L}$. And so on for any other specific multiplier. In general, if the vector variable $\mathbf{y}$ is a magnitude of interest and the ratios of $\mathbf{y}$ to $\mathbf{x}$ are known, that is:

$$
\mathbf{y}_{\mathbf{c}}^{\prime}=\mathbf{y}^{\prime} \cdot\langle\mathbf{x}\rangle^{-1}
$$


with $\langle\mathbf{x}\rangle$ representing the diagonal matrix reproducing the coefficients in vector $\mathbf{x}$, then the $\mathbf{y}$ multipliers will be given by:

$$
\mathbf{m}_{y}^{\prime}=\mathbf{y}_{\mathbf{c}}^{\prime} \cdot \mathbf{L}
$$

Oftentimes the interest lies in presenting detailed bilateral multiplier data. This requires the use of matrices. For output multipliers Definition 1 yields that the output multiplier matrix, which we will denote as $\mathbf{M}_{x}$, is in fact $\mathbf{L}$ :

$$
\mathbf{M}_{x}=\mathbf{L}
$$

For value-added multipliers, using Definition 2, the multiplier matrix will be:

$$
\mathbf{M}_{v}=\left\langle\mathbf{v}_{\mathbf{c}}\right\rangle \cdot \mathbf{L}
$$

with $\left\langle\mathbf{v}_{\mathbf{c}}\right\rangle$ being the diagonalised version of the vector of ratios of value-added over total output. In general for a magnitude of interest $\mathbf{y}$, the corresponding multiplier matrix is given by:

$$
\mathbf{M}_{y}=\left\langle\mathbf{y}_{\mathbf{c}}\right\rangle \cdot \mathbf{L}
$$

Of course, expression (15) can be rewritten into the general format in (17) simply by:

$$
\mathbf{M}_{x}=\langle\mathbf{e}\rangle \cdot \mathbf{L}
$$

The relationship between multiplier vectors and multiplier matrices is immediate. For the general case of given magnitude $\mathbf{y}$ :

$$
\mathbf{m}_{y}^{\prime}=\mathbf{e}^{\prime} \cdot \mathbf{M}_{y}=\mathbf{e}^{\prime} \cdot\left\langle\mathbf{y}_{\mathbf{c}}\right\rangle \cdot \mathbf{L}
$$

In general terms, all that is needed to derive a multiplier vector $\mathbf{m}_{y}^{\prime}$ and a multiplier matrix $\mathbf{M}_{y}$ is the technological information on coefficients contained in matrix $\mathbf{A}$ and vector $\mathbf{y}_{\mathbf{c}}^{\prime}$.

The fact that in actual practice, input-output value data is used as if that data were as well physical data (the above-mentioned normalisation) has important formal and content implications. 
Proposition 1: In the standard implementation of the input-output model, i.e. the Leontief model, all value-added multipliers are unitary. Therefore the 'conservancy of value' rule is verified.

Proof: From expressions (6) and (7) it follows that $\mathbf{v}_{\mathbf{c}}^{\prime} \cdot \mathbf{L} \cdot \mathbf{f}=\mathbf{e}^{\prime} \cdot \mathbf{f}$. Differentiate the left-hand side to obtain value-added multipliers, and the right hand side to obtain the unit vector, i.e. $\mathbf{m}_{v}^{\prime}=\mathbf{e}^{\prime}$. QED.

When an extra unit of demand for $j$, with a currency value of one, is injected into the economy, total additional income - as measured by value-added changes in all sectors - increases exactly by one unit of value. This makes perfect sense since in a static model with no technical progress there cannot be endogenous 'growth'. Value injected (i.e. 1) in $j$ is exactly equal to value created (i.e. 1) in the economy although its sectoral distribution $\left(v_{c i} \cdot \ell_{i j}\right)$ will be unequally distributed, depending on the peculiarities of the technology ${ }^{3}$.

OS criticize standard output multipliers for their 'overestimation' of the economic impact of exogenous unitary injections. Quantitatively they are right. If we look at the series expansion of the Leontief inverse:

$$
\mathbf{L}=\mathbf{I}+\mathbf{A}+\mathbf{A}^{2}+\mathbf{A}^{3}+\cdots+\mathbf{A}^{\mathbf{k}}+\cdots
$$

the unitary injections are picked up by the first term $\mathbf{I}$ of the right-hand side. Because matrix $\mathbf{A}$ is non-negative, it is trivial that $\mathbf{L} \geq \mathbf{I}$, i.e. output multiplier effects cannot be smaller than unity: $\mathbf{m}_{x}^{\prime}=\mathbf{e}^{\prime} \cdot \mathbf{L} \geq \mathbf{e}^{\prime}$. Is this a problem for standard output multipliers? According to OS it is. They state:

"Naturally, this cannot be true. When the claims of all sectors in the economy are added an (implicit) estimate of the total size of the economy will result that is many times larger than its actual size". (op. cit, 2002).

But this is a problem only if we fail to remember that intermediate transactions are netted out in national accounting, precisely, to avoid double counting. Total (or 'gross') output is not a

\footnotetext{
${ }^{3}$ If we could distinguish between value and actual physical data, the same conservation of value property would hold but in that case we would need to introduce non-unitary prices explicitly. With the implicit normalisation developments are simpler and clearer.
} 
magnitude of interest in the National and Income Product Accounts. Only final (or 'net') output is of interest as far as the proper accounting of economic variables is concerned. Their statement is quantitatively correct but, regarding national accounting rules, conceptually irrelevant. It does not matter if output multipliers are, in general, greater than unity nor this violates any economic or accounting laws. This fact only reflects the extent of the indirect general equilibrium effects in production, information that can be indeed relevant for many purposes, but bears no illconceptual or methodological — in itself. A new unitary injection of final demand will always generate a unit of new value, provided we stay away from the malpractice of double counting.

A new policy or project that entails changes in final demand, say by $\Delta \mathbf{f}$, will have an economy-wide output impact that can be estimated, under the assumptions of the interindustry model, by $\mathbf{m}_{x}^{\prime} \cdot \Delta \mathbf{f}$ if we are interested in the total output effect. If aggregate sectoral effects are also of interest, then we can use $\mathbf{m}_{x}^{\prime} \cdot\langle\boldsymbol{\Delta} \mathbf{f}\rangle$ to disaggregate the total effect. Finally, bilateral sectorby-sector effects can be obtained using $\mathbf{M}_{x} \cdot\langle\boldsymbol{\Delta} \mathbf{f}\rangle$. Similarly, for value-added we would use the value-added multiplier information and would therefore estimate effects by, respectively, magnitudes $\mathbf{m}_{v}^{\prime} \cdot \Delta \mathbf{f}, \mathbf{m}_{v}^{\prime} \cdot\langle\Delta \mathbf{f}\rangle$ and $\mathbf{M}_{v} \cdot\langle\Delta \mathbf{f}\rangle$.

OS correctly point out that, instead, practitioners commonly but mistakenly use estimated changes in production $\Delta \mathbf{x}$ (or value-added $\Delta \mathbf{v}$ ) to evaluate the impact of a policy. For instance, using as output estimates $\mathbf{m}_{x}^{\prime} \cdot \Delta \mathbf{x}$ (or related magnitudes $\mathbf{m}_{x}^{\prime} \cdot\langle\Delta \mathbf{x}\rangle$ or $\mathbf{M}_{x} \cdot\langle\Delta \mathbf{x}\rangle$ ) there is no doubt whatsoever that double counting is surely and erroneously introduced. What drives the interindustry model is exogenous final demand $\Delta \mathbf{f}$ not endogenous total output $\Delta \mathbf{x}$. Their proposed remedy is to substitute standard 'gross' multipliers for their new 'net' multipliers, which filter through the overestimation resulting from the erroneous use of magnitudes such as $\Delta \mathbf{x}$. With some slight notational modifications regarding their own definitions, OS's description is as follows.

Definition 3: 'Net' output multipliers. Denoted by $\boldsymbol{\mu}_{x}^{\prime}$, they are the standard output multipliers corrected by the filtering vector $\mathbf{f}_{\mathbf{c}}$ of ratios of exogenous final demand to total output, i.e. $\mathbf{f}_{\mathbf{c}}=\langle\mathbf{x}\rangle^{-1} \cdot \mathbf{f}$. From here:

$$
\boldsymbol{\mu}_{x}^{\prime}=\mathbf{m}_{x}^{\prime} \cdot\left\langle\mathbf{f}_{\mathbf{c}}\right\rangle=\mathbf{e}^{\prime} \cdot \mathbf{L} \cdot\left\langle\mathbf{f}_{\mathbf{c}}\right\rangle=\mathbf{e}^{\prime} \cdot \mathbf{L} \cdot\langle\mathbf{e}\rangle^{-1} \cdot\left\langle\mathbf{f}_{\mathbf{c}}\right\rangle
$$


With this definition, regular output multipliers are filtered through the ratios $\mathbf{f}_{\mathbf{c}}$ and are (trivially standardised) by the inverse of the diagonalised output-to-output ratios $\langle\mathbf{e}\rangle$. The filtering by $\mathbf{f}_{\mathbf{c}}$ ensures that only the exogenous part of total output is taken into account. Similarly, we also have:

Definition 4: 'Net' value-added multipliers. Denoted by $\boldsymbol{\mu}_{v}^{\prime}$, they are the standard value-added multipliers corrected by the filtering vector $\mathbf{f}_{\mathbf{c}}$ and standardised by the inverse of the diagonalised value-added to total output ratios $\left\langle\mathbf{v}_{\mathbf{c}}\right\rangle$ :

$$
\boldsymbol{\mu}_{v}^{\prime}=\mathbf{m}_{v}^{\prime} \cdot\left\langle\mathbf{v}_{\mathbf{c}}\right\rangle^{-1}\left\langle\mathbf{f}_{\mathbf{c}}\right\rangle=\mathbf{v}_{\mathbf{c}}^{\prime} \cdot \mathbf{L} \cdot\left\langle\mathbf{v}_{\mathbf{c}}\right\rangle^{-1}\left\langle\mathbf{f}_{\mathbf{c}}\right\rangle
$$

Once again, we can check the nice formal similarity in the multipliers structure. In general, for any magnitude of interest $\mathbf{y}$, and using (13) and (14), we would derive the mapping transforming 'gross' into 'net' multipliers as:

$$
\mathbf{m}_{y}^{\prime} \rightarrow \boldsymbol{\mu}_{y}^{\prime}=\mathbf{m}_{y}^{\prime} \cdot\left\langle\mathbf{y}_{\mathbf{c}}\right\rangle^{-1}\left\langle\mathbf{f}_{\mathbf{c}}\right\rangle=\mathbf{y}_{\mathbf{c}}^{\prime} \cdot \mathbf{L} \cdot\left\langle\mathbf{y}_{\mathbf{c}}\right\rangle^{-1}\left\langle\mathbf{f}_{\mathbf{c}}\right\rangle
$$

We now proceed to discuss why the 'net' multiplier concept of OS does not have a sound theoretical basis nor actually performs as a multiplier.

\section{THE TROUBLE WITH OS ‘NET’ MULTIPLIERS.}

OS state that "the reason for developing the concept of the net multiplier" is their first theorem, which we reproduce here for completeness's sake:

(OS) Theorem 1: The output-weighted average of all sectoral net multipliers equals unity.

The following counterexample, however, shows the statement to be false. From the fictitious but completely regular input-output data in Table 1 of the Appendix we compute standard and 'net' multipliers. From the table we compute all the relevant and needed information:

$$
\text { Leontief inverse: } \mathbf{L}=\left[\begin{array}{lll}
1.583 & 0.522 & 0.719 \\
0.791 & 1.511 & 0.360 \\
1.223 & 0.971 & 2.374
\end{array}\right]
$$


Value-added coefficients: $\mathbf{v}_{\mathbf{c}}^{\prime}=\left(\begin{array}{lll}0.2 & 0.4 & 0.3\end{array}\right)$

Filtering vector: $\mathbf{f}_{\mathbf{c}}=\left[\begin{array}{c}0.5 \\ 0.4 \\ 0\end{array}\right]$

The reader can now verify these multiplier values to be correct:

$$
\begin{aligned}
& \mathbf{m}_{x}^{\prime}=\left(\begin{array}{lll}
3.597 & 3.004 & 3.453
\end{array}\right) \\
& \mathbf{m}_{v}^{\prime}=\left(\begin{array}{lll}
1 & 1 & 1
\end{array}\right) \\
& \boldsymbol{\mu}_{x}^{\prime}=\left(\begin{array}{lll}
1.799 & 1.201 & 0
\end{array}\right) \\
& \boldsymbol{\mu}_{v}^{\prime}=\left(\begin{array}{lll}
2.5 & 1 & 0
\end{array}\right)
\end{aligned}
$$

However, it turns out that for the output vector $\mathbf{x}$ in the table and total aggregate output $x=\mathbf{e}^{\prime} \cdot \mathbf{x}$ we obtain:

$$
\boldsymbol{\mu}_{v}^{\prime} \cdot \mathbf{x} \cdot x^{-1}=1.167 \neq 1
$$

which shows the statement in Theorem 1 not to be true. The fact that $\boldsymbol{\mu}_{v}^{\prime} \cdot \mathbf{x} \cdot x^{-1} \neq 1$ indicates that 'net' value-added multipliers do not satisfy the 'conservation of value' property commented above. The example, in addition, is fully conventional and completely non-pathological ${ }^{4}$. The reader can construct as many different examples as he or she wishes. So, what gives?

In OS's proof, the logical chain of reasoning fails when they inadvertently take the relationship $\mathbf{v}_{\mathbf{c}}^{\prime} \cdot \mathbf{L} \cdot\langle\mathbf{f}\rangle=\mathbf{v}^{\prime}$ as true when in fact is false. Indeed, in Proposition 1 above we have shown that $\mathbf{v}_{\mathbf{c}}^{\prime} \cdot \mathbf{L}=\mathbf{e}^{\prime}$, therefore $\mathbf{v}_{\mathbf{c}}^{\prime} \cdot \mathbf{L} \cdot\langle\mathbf{f}\rangle=\mathbf{e}^{\prime} \cdot\langle\mathbf{f}\rangle=\mathbf{f}^{\prime}$ and their step in the demonstration is clearly faulty since in general there is no empirical or theoretical reason whatsoever for $\mathbf{f}^{\prime} \cdot\langle\mathbf{v}\rangle^{-1}$ to be the unitary vector OS need in their proof.

\footnotetext{
${ }^{4}$ Pathological counterexamples can sometimes be avoided by strengthening the set of assumptions in the initial statement, giving rise, in these cases, to a stronger mathematical proposition. Non-pathological counterexamples, however, are harder to crack since they usually indicate a serious logical or reasoning flaw.
} 
The correction by the filtering vector $\mathbf{f}_{\mathbf{c}}$ is $a d-h o c$ and can be seen to yield a simple accounting reshuffling of the initial data that compensates ${ }^{5}$ for the improper combined use of 'gross' multipliers and gross output. That this is the case can be seen in the following result, which also shows that equilibrium changes will break down the reshuffling rule.

Proposition 2: Initial data on output and final demand satisfying the equilibrium conditions - and only these initial data-can be reshuffled using 'net' and 'gross' output multipliers. In other words: (i) $\boldsymbol{\mu}_{x}^{\prime} \cdot\langle\mathbf{x}\rangle=\mathbf{m}_{x}^{\prime} \cdot\langle\mathbf{f}\rangle$ (ii) $\boldsymbol{\mu}_{x}^{\prime} \cdot \mathbf{x}=\mathbf{m}_{x}^{\prime} \cdot \mathbf{f}$, but in general terms (iii) $\boldsymbol{\mu}_{x}^{\prime} \cdot \Delta \mathbf{x} \neq \mathbf{m}_{x}^{\prime} \cdot \mathbf{\Delta} \mathbf{f}$.

Proof: (i) From Definition 3 we obtain:

$$
\boldsymbol{\mu}_{x}^{\prime} \cdot\langle\mathbf{x}\rangle=\mathbf{m}_{x}^{\prime} \cdot\left\langle\mathbf{f}_{\mathbf{c}}\right\rangle \cdot\langle\mathbf{x}\rangle=\mathbf{m}_{x}^{\prime} \cdot\langle\mathbf{f}\rangle \cdot\langle\mathbf{x}\rangle^{-1} \cdot\langle\mathbf{x}\rangle=\mathbf{m}_{x}^{\prime} \cdot\langle\mathbf{f}\rangle
$$

(ii) follows from aggregation of the result in (i) since $\langle\mathbf{x}\rangle \cdot \mathbf{e}=\mathbf{x}$, and likewise $\langle\mathbf{f}\rangle \cdot \mathbf{e}=\mathbf{f}$. As for (iii) suppose the condition were to hold in equilibrium, i.e. $\boldsymbol{\mu}_{x}^{\prime} \cdot \Delta \mathbf{x}=\mathbf{m}_{x}^{\prime} \cdot \boldsymbol{\Delta f}$. Substituting we would find $\mathbf{m}_{x}^{\prime} \cdot\left\langle\mathbf{f}_{\mathbf{c}}\right\rangle \cdot \Delta \mathbf{x}=\mathbf{m}_{x}^{\prime} \cdot \Delta \mathbf{f}$ and from here $\left\langle\mathbf{f}_{\mathbf{c}}\right\rangle \cdot \Delta \mathbf{x}=\Delta \mathbf{f}$. This would imply for all $j$ that $\left(f_{j} / x_{j}\right) \cdot \Delta x_{j}=\Delta f_{j}$ or with a slight rearrangement $\left(\Delta x_{j} / x_{j}\right) /\left(\Delta f_{j} / f_{j}\right)=1$. These ratios are nothing but the output elasticities to own final demand. In conclusion, all these elasticities would be unitary. This can be seen to be a general contradiction to the equilibrium condition $\mathbf{x}=\mathbf{L} \cdot \mathbf{f}$. Indeed, from this condition we can see immediately that $x_{j}=\sum_{i} \ell_{i j} \cdot f_{i}$ and $\Delta x_{j} / \Delta f_{j}=\ell_{i j}$. We label now the output elasticity to own final demand by $\varepsilon_{j}$. Then one can quickly check:

$$
\varepsilon_{j}=\frac{\left(\Delta x_{j} / x_{j}\right)}{\left(\Delta f_{j} / f_{j}\right)}=\frac{\left(\Delta x_{j} / \Delta f_{j}\right)}{\left(x_{j} / f_{j}\right)}=\frac{\ell_{j j}}{\left(\sum_{i} \ell_{i j} \cdot f_{i}\right) / f_{j}}=\frac{\ell_{j j} \cdot f_{j}}{\sum_{i} \ell_{i j} \cdot f_{i}}<1
$$

as long as $\ell_{i j}>0$, which is the common, standard situation for the Leontief inverse matrix $\mathbf{L}$. QED.

This result tells us that OS 'net' multipliers do compensate for double counting but they do so only for the initial equilibrium. Under standard interindustry characteristics, changes that satisfy

\footnotetext{
${ }^{5}$ Although the idea is of course contained in Oosterhaven \& Stelder (2002), the first explicit reference to OS 'net' multipliers as compensators for double counting is in De Mesnard (2007a).
} 
the equilibrium conditions of the model will fail to satisfy the compensating property. De Mesnard (2007a) also points out in his Theorem 1 that the filtering vector $\mathbf{f}_{\mathbf{c}}$ cannot be stable. Notice however that in a very peculiar-and implausible-case stability would ensue, i.e. $\varepsilon_{j}=1$, provided all $\ell_{i j}=0$ for $i \neq j$ and $\ell_{j j}>0$. This case can be seen to correspond to a degenerate economy with fully autarkic production in which no sector relates to any other sectors except itself. We explore this circumstance in the next formal result, which clarifies, complements, and strengthens some of De Mesnard's remarks.

Proposition 3: Let $\mathbf{A} \geq 0$ be a productive interindustry matrix such that $a_{i j}=0$ for $i \neq j$ and $a_{i i}>0$. Then all output elasticities $\mathcal{\varepsilon}_{j}$ are unitary.

Proof: Since matrix $\mathbf{A}$ is diagonal all its powers $\mathbf{A}^{k}$ will be diagonal too with their generic elements being $\left(a_{j j}\right)^{k}$. Since $\mathbf{A}$ is productive, we will have $a_{j j}<1$. Also, expression (20) holds and we can compute convergence simply by the individual diagonal series. In this case $\mathbf{L}$ will be diagonal too and its generic element is given by the geometric sum of the diagonal elements, i.e. $\ell_{j j}=\sum_{k=0}^{\infty}\left(a_{j j}\right)^{k}=\left(1-a_{j j}\right)^{-1}$. Using the equilibrium condition (5) it is immediate in this case that, for all $j, x_{j}=\ell_{j j} \cdot f_{j}$. From here it follows that $\Delta x_{j}=\ell_{i j} \cdot \Delta f_{j}$ and $\varepsilon_{j}=1$. QED.

If the economy were fully sector autarkic, as in Proposition 3, then the filtering vector $\mathbf{f}_{\mathbf{c}}$ would be invariant over different equilibria and the 'net' multiplier concept would have accounting consistency and be invariant too. But this type of economies is empirically implausible and lacks realism. Simply stated, they do not seem to exist in the real world and they are of little theoretical interest for quantitative modelling and analysis.

The 'net' multiplier concept, in addition, cannot be used as a predictor of change, as regular multipliers can - at least within the confines of the interindustry model assumptions. Let us take again the numerical example above. We observe that 'net' output and value-added multiplier values for good 3 are both nil, i.e. $\mu_{x, 3}=0$ and $\mu_{v, 3}=0$. Clearly these values cannot be interpreted as predictors for change; in fact, a literal reading should predict zero effect no matter what the exogenous change is. We know this cannot be true as simple examples would again show. But conceptually a multiplier is nothing but a normalised evaluation of a unitary external change. This is the reason we can predict that if we double the change, then we double the estimated effect — of course and again under the restrictions imposed by the interindustry model assumptions. The 
above example values for OS 'net' multipliers, however, predict no change, regardless of the size of the new impulse. This simply cannot be. It is clear the culprit, so to speak, is the fact that final demand for good 3 is zero. This makes the third component in the filtering vector to be zero. Standard multipliers, in contrast, are unaffected by final demand being zero, as it of course should be.

Notice, also from the example, another perplexing observation on 'net' value-added multipliers, namely, they coincide with the direct ratios of final demand to value-added, i.e. $\mu_{v, j}=f_{j} / v_{j}$. This is no numerical coincidence as the next straightforward result illustrates.

Proposition 4: 'Net' value-added multipliers do not amplify any initial impulses and they simply reproduce the direct sectoral ratios of final demand to value-added from the initial data. In other words, $\boldsymbol{\mu}_{v}^{\prime}=\mathbf{f}^{\prime} \cdot\langle\mathbf{v}\rangle^{-1}$.

Proof: From Definition 4 and Proposition 1 we can write:

$$
\boldsymbol{\mu}_{v}^{\prime}=\mathbf{v}_{\mathbf{c}}^{\prime} \cdot \mathbf{L} \cdot\left\langle\mathbf{v}_{\mathbf{c}}\right\rangle^{-1} \cdot\left\langle\mathbf{f}_{\mathbf{c}}\right\rangle=\mathbf{e}^{\prime} \cdot\left\langle\mathbf{v}_{\mathbf{c}}\right\rangle^{-1} \cdot\left\langle\mathbf{f}_{\mathbf{c}}\right\rangle=\mathbf{e}^{\prime} \cdot\langle\mathbf{v}\rangle^{-1}\langle\mathbf{x}\rangle \cdot\langle\mathbf{x}\rangle^{-1} \cdot\langle\mathbf{f}\rangle=\mathbf{e}^{\prime} \cdot\langle\mathbf{v}\rangle^{-1} \cdot\langle\mathbf{f}\rangle
$$

We can now freely reorder the presence of the diagonal matrices to obtain the sought result:

$$
\boldsymbol{\mu}_{v}^{\prime}=\mathbf{e}^{\prime} \cdot\langle\mathbf{v}\rangle^{-1} \cdot\langle\mathbf{f}\rangle=\mathbf{e}^{\prime} \cdot\langle\mathbf{f}\rangle \cdot\langle\mathbf{v}\rangle^{-1}=\mathbf{f}^{\prime} \cdot\langle\mathbf{v}\rangle^{-1} \quad \text { QED. }
$$

The essence of multipliers is that they capture economy-wide interdependence effects and by doing so they modify the initial direct ratios that are constructed from the interindustry data. The modification works by adding up the direct and indirect effects. Under this light, OS 'net' value-added multipliers do not seem to be capturing anything inherent to the set of adjustments working to lead the economy to a new equilibrium; on the contrary, they merely reproduce initial data ratios. This is very damaging indeed for the 'net' multiplier concept for the same ratios would keep appearing for completely different interindustry data and technology. See for instance Table 2 in the Appendix, where an alternative interindustry data is presented which shares the same $\mathbf{v}^{\prime}, \mathbf{f}$ and $\mathbf{x}$ with the data in Table 1 . The technology matrix $\mathbf{A}$ is visibly different from the previous one from Table 1, and thus so will be the inverse $\mathbf{L}$. However, the 'net' value-added multipliers can be seen to be exactly the same as before. 'Net' value-added multipliers are independent of the technology, a surprising and undesirable trait, since technology should be the leading force in 
driving all type of multiplier effects. Even more, this technological duplicity yielding the same value-added 'net' multipliers is the rule rather than the exception. It is not a curiosum, as it rests upon the fact that technology has nothing to do with 'net' value-added multipliers as we have seen in Proposition 4. The following Corollary provides some additional formal support to this result.

Corollary (Multiplicity): Given any economy $(\mathbf{Z}, \mathbf{v}$ ',f) with 'net' value-added multipliers $\boldsymbol{\mu}_{v}^{\prime}$, there are infinitely many alternative and different in technology economies $\left(\breve{\mathbf{Z}}, \mathbf{v}^{\prime}, \mathbf{f}\right)$ producing exactly the same 'net' value-added multipliers.

Proof: For an algebraic confirmation of the Corollary, take any positive real number $\breve{z}$ and subtract it from entry $z_{i j}$ in position $(i, j)$ of matrix $\mathbf{Z}$, i.e. $\breve{z}_{i j}=z_{i j}-\breve{z}$. Add $\breve{z}$ to the entry in position $\left(i, j^{\prime}\right)$ with $j^{\prime} \neq j$, subtract it from the entry in position $\left(i^{\prime}, j^{\prime}\right)$ with $i^{\prime} \neq i$ and finally add it up to position $\left(i^{\prime}, j\right)$. These changes transform the flows in $\mathbf{Z}$ and generate a new and different $\breve{\mathbf{Z}}$ but leave vectors $\mathbf{x}, \mathbf{v}^{\prime}$ and $\mathbf{f}$ invariant. Therefore there are infinitely many technology matrices $\breve{\mathbf{A}}=\breve{\mathbf{Z}} \cdot\langle\mathbf{x}\rangle^{-1}$ for the same multiplier values, one for each possible real number $\breve{z}>0$. QED.

Let us recapitulate. In addition to De Mesnard's criticism on the empirical instability of 'net' multipliers, we have reached these novel and conceptually damaging conclusions: (i) theorems that should give theoretical support to the OS 'net' multiplier concept have been shown to be generically untrue. Additionally, (ii) OS 'net' multipliers cannot be interpreted as partial derivatives within the causal logic of an economic model or its mathematical counterpart; (iii) they cannot be used as sensible predictors for change except in empirically unrealistic economies; (iv) their values can be totally blind to the underlying technology. We believe all these reasons indicate it is time for the OS concept to be discarded as unfit for performing rigorous economic analysis.

\section{SOME REFLECTIONS: WHAT ARE WE LEFT WITH?}

At this juncture three possibilities can be considered. The first one is to refine the 'net' multiplier concept. The second one consists in trying to redefine the idea. The final possibility is to abandon altogether the 'net' approach, stick with the standard 'gross' multipliers and use them judiciously, of course.

De Mesnard's (2002, 2007a, 2007b) alternative 'net' multiplier concept and Diezenbacher's (2005) ingenious and original reinterpretation are attempts to take the refinement 
road. Omitting here the technical details, De Mesnard's own 'net' output multiplier concept is seen to be defined by:

$$
\boldsymbol{\eta}_{x}^{\prime}=\mathbf{e}^{\prime} \cdot \mathbf{L} \cdot \mathbf{A}
$$

and satisfies the following linear relationship with standard 'gross' multipliers:

$$
\mathbf{m}_{x}^{\prime}=\boldsymbol{\eta}^{\prime}(\mathbf{x})+\mathbf{e}^{\prime}
$$

Unlike OS multipliers, De Mesnard's have the nice property of being invariant, a property they share with standard 'gross' multipliers. Unlike OS proposal, however, De Mesnard's 'net' multipliers only partially compensate for double counting, even at the initial data set, as a simple numerical calculation using the data in Table 1 and the example above proves. Recall that compensating for the malpractice of double counting was precisely OS's goal in developing their 'net' multiplier proposal. The economic content of De Mesnard's 'net' multipliers can be seen immediately from a slight rearrangement of (24):

$$
\eta^{\prime}(x)=e^{\prime} \cdot \mathbf{L} \cdot A=e^{\prime} \cdot\left(I+A+A^{2}+A^{3}+\cdots\right) \cdot A=e^{\prime} \cdot\left(A+A^{2}+A^{3}+\cdots\right)
$$

This version of 'net' multipliers is therefore based on the sum of all of the rounds of productive impulses that accumulate in response to exogenous unitary injections. On the other hand, these cumulative, indirect effects are catalogued as part of the 'gross' output of an economy from the perspective of the National Income and Product Accounts and they are systematically netted out to steer clear of double counting. Behind De Mesnard's 'net' multipliers we find precisely what, in fact, makes standard interindustry multipliers to be considered as measures of 'gross' output. They are indicators of the intermediate output response to exogenous injections in final demand, that is, in final or 'net' output. Intermediate production is given by $\mathbf{A} \cdot \mathbf{x}$ while 'gross' output is obtained from $\mathbf{x}=\left(\mathbf{I}+\mathbf{A}+\mathbf{A}^{2}+\cdots\right) \cdot \mathbf{f}$, hence:

$$
A \cdot \mathbf{x}=\mathbf{A} \cdot\left(\mathbf{I}+\mathbf{A}+\mathbf{A}^{2}+\cdots\right) \cdot \mathbf{f}=\mathbf{A} \cdot \mathbf{L} \cdot \mathbf{f}=\mathbf{L} \cdot \mathbf{A} \cdot \mathbf{f}
$$

Let us now call $\mathbf{q}^{\prime}$ to the vector of intermediate unitary outputs, i.e. $\mathbf{q}^{\prime}=\mathbf{e}^{\prime} \cdot \mathbf{A}$ where each component $q_{j}$ measures total intermediate outputs of all goods that are required to produce one unit of 'gross' output $j$. Aggregating by columns and denoting by $q$ total intermediate output we obtain from expression (27): 


$$
q=\mathbf{q}^{\prime} \cdot \mathbf{x}=\mathbf{e}^{\prime} \cdot \mathbf{A} \cdot \mathbf{x}=\mathbf{e}^{\prime} \cdot \mathbf{L} \cdot \mathbf{A} \cdot \mathbf{f}=\boldsymbol{\eta}_{x}^{\prime} \cdot \mathbf{f}
$$

Taking derivatives in (28) we find:

$$
\frac{d q}{d f_{j}}=\eta_{x, j}
$$

From expression (29) we can see that De Mesnard's 'net' multipliers are actually measuring an effect on 'gross' output resulting from unitary injections in final demand - but on the intermediate output part, exclusively. It is worth recalling that this part is endogenously determined in the standard Leontief model. In other words, this 'net' multiplier is, in its roots, as standard as any traditional 'gross' multiplier, those that are evaluated from impulses starting in final demand, but provides just a truncated measure of the effects on 'gross' output.

Certainly something does not quite add up here and it is probably more than just denominational divergence. Much of the conceptual difficulty stems, we believe, from stretching the nature and intrinsic properties of the basic interindustry model. The interindustry model is represented by equation (5), which thanks to its linearity can be used either in absolute levels or in differential terms. It is customary in economics to take vector variable $\mathbf{f}$ ( or $\boldsymbol{\Delta} \mathbf{f}$ ) as exogenous and vector variable $\mathbf{x}$ (or $\Delta \mathbf{x}$ ) as endogenous. For this direction of causality, matrix $\mathbf{L}$ is in fact the Jacobian matrix of the model. As such, it measures the sensitivity of the endogenous solution to the exogenous variables or, in calculus terminology, the partial derivatives of each type of output (dependent variable) with respect to every type of final demand (independent variable). There is however plenty of mathematical freedom to modify the classification of endogenous (dependent) and exogenous (independent) variables. We may want to study the equilibrium effects of a change in some level of 'gross' output, but in this case a previously exogenous variable (in the final demand vector) imperatively needs to become endogenous to maintain the mathematical consistency (e.g. $n$ equations, $n$ unknowns) of the system of equations representing the economic model. From the causality viewpoint, however, it is not easy to find a justification for which final demand will become endogenous. Mathematically speaking, any $f_{j}$ can be made endogenous and the model will still be solvable for the new classification of variables. What is certainly not possible is for a variable in the interindustry equation to be at the same time endogenous and exogenous. This leads to hard to fathom conceptual, logical and interpretative issues within the interindustry model, as we have seen in the previous discussion on 'net' multipliers. If we depart 
from a causal or modelling perspective and keep the analysis within a pure accounting angle, it turns out that from a reinterpretation of the Hawkins-Simon condition the accounting effect of gross output on gross output can be numerically quantified. See Gim \& Kim (1989) and Sancho (2012) for some developments along this line.

De Mesnard's driving idea for his 'net' multipliers is to evaluate the impact on all sectors' output as a result of a particular industry deciding to exogenously increase its total output. This entails that such a decision is indeed exogenous and can be effectively taken. If it cannot be taken, the issue looses interest, both from modelling and policy perspectives. If on the contrary the decision can be taken, then such an industry would not be behaving competitively, a basic principle of the interindustry model where no sector has market power and all industries are pricetakers. Under non-competitive behaviour, De Mesnard's point would indeed become quite relevant but then other type of assumptions and models, fairly different from those of the competitive interindustry model, would be required.

If refining the 'net' multiplier concept seems difficult or more plainly inappropriate, a second way to proceed is to redefine the notion. We mean here a change in emphasis from 'net' multipliers to 'net multipliers', that is, focusing the attention on multipliers that try to measure 'net effects' on output. This is equivalent to measure effects on final or net production, or in GDP. This would have the advantage of being conformal with the typical measurements in the National Income and Product Accounts. In the interindustry model, at least in the standard implementation that has been used in the 'net' multipliers literature, new net output coincides exactly with the external injections from final demand. Output 'net multipliers', in this case, would therefore have trivial numerical values. Perhaps the option is to explore multipliers in other type of models where net production, or GDP, follows interaction rules different from those of the interindustry model. We are specifically thinking of applied general equilibrium models where, in principle, multipliers for both total ('gross') output and final ('net') output are relatively easy to estimate using the Jacobian of the non-linear general equilibrium model.

Back to the interindustry model, and when all things are considered, we believe that using standard multipliers is a safe bet since they satisfy all the required conceptual, mathematical and interpretative conditions. Properly used they respect the conservancy of value rule, are built from the Jacobian matrix of the model's equation, have clear economic meaning and can therefore be used as predictors for change. If they do the job, do we really need something else? 


\section{CONCLUDING REMARKS}

OS correctly pointed out the misuse of the interindustry model and its standard multipliers in professional practice and consultancy. This malpractice, leading to economic double counting of endogenous effects and its corresponding upward bias in performing impact or policy evaluations, cannot be accepted on scientific grounds. OS should be commended for denouncing it and bringing its erroneous nature to the public fore. Their therapy, however, does not solve the problem and introduces further and probably unsolvable difficulties of a theoretical nature. Suggesting a way out that can be quickly, and wrongly, interpreted by practitioners as a justification for their malpractice will only compound and aggravate the problem. Clearly, OS do not condone the misuse of the model and do not suggest the continuance of the same bad practices, but careless professionals may feel nonetheless justified and thus keep doing their business as usual. Bad practices should always be chastised. If smoking is bad, the therapy is to stop smoking - hard as it may be; smoking with a filter does not solve the problem and may in fact, out of a false sense of security, make the habit persistent or even more intense.

\section{Acknowledgements}

Support from research projects MICINN-ECO2009-11857 and SGR2009-578 is gratefully acknowledged. Clarifying comments by L. Alsedà and thorough discussions with A. Manresa are very much appreciated. The usual caveat applies.

\section{REFERENCES}

De Mesnard, L. (2002). "Note about the Concept of Net Multipliers”. Journal of Regional Science, vol. 42(3), pp. 545-548.

De Mesnard, L. (2007a). “A critical comment on Oosterhaven-Stelder net multipliers”. Annals of Regional Science, vol. 41, pp. 249-271.

De Mesnard, L. (2007b). "Reply to Oosterhaven: the net multiplier is a new key sector indicator". Annals of Regional Science, vol. 41, pp. 285-296.

Dietzenbacher, E. (2005). "More on Multipliers”. Journal of Regional Science, vol. 45(2), pp. 421426. 
Gim, H. U. \& Kim, K. (1998). "The general relation between two different notions of direct and indirect input requirements". Journal of Macroeconomics, vol. 20(1), pp. 199-208.

Leontief, W. (1936). "Quantitative input-output relations in the economic system of the United States". Review of Economics and Statistics, vol. 18(3), pp. 105-125.

Márquez, M.A. (2011). “Centralidad e integración de las redes económicas de los sectores de alta tecnología en América del Norte", UNAM working paper.

Nikaido, H. (1972). Introduction to Sets and Mappings in Modern Economics. North-Holland, Amsterdam, the Netherlands.

Oosterhaven, J. \& Stelder, D. (2002). “Net Multipliers Avoid Exaggerating Impacts: With a BiRegional Illustration for the Dutch Transportation Sector,' Journal of Regional Science, vol. 42(3), pp. 533-543.

Oosterhaven, J. (2004). "On the definition of key sectors and the stability of net versus gross multipliers". Research report 04C01, SOM Research School, Groningen University.

Oosterhaven, J. (2007). "The net multiplier is a new key sector indicator: reply to De Mesnard's comment”. Annals of Regional Science, vol. 41, pp. 273-283.

Sancho, F. (2012). "Straightening out the concept of direct and indirect input requirements". Economics Bulletin, vol. 32(1), pp. 502-509.

Temurshoev, U. \& Oosterhaven, J. (2010). “On input-output linkage measures”. Working papers in input-output economics, WPIOX 10-002. 


\section{Appendix}

Table 1: $\quad$ Base interindustry data

\begin{tabular}{|c|ccc|c|c|}
\hline $\mathbf{Z}$ & 1 & 2 & 3 & $\mathbf{f}$ & $\mathbf{x}$ \\
\hline 1 & 10 & 15 & 25 & 50 & 100 \\
2 & 40 & 20 & 0 & 40 & 100 \\
3 & 30 & 25 & 45 & 0 & 100 \\
\hline $\mathbf{v}^{\prime}$ & 20 & 40 & 30 & \multicolumn{1}{|c}{} \\
\cline { 1 - 3 } $\mathbf{x}^{\prime}$ & 100 & 100 & 100 & & \\
\cline { 1 - 2 } & &
\end{tabular}

Table 2: $\quad$ Alternate interindustry data

\begin{tabular}{|c|c|c|c|c|c|}
\hline Ž & 1 & 2 & 3 & f & $\mathbf{x}$ \\
\hline 1 & 10 & 20 & 20 & 50 & 100 \\
\hline 2 & 30 & 0 & 30 & 40 & 100 \\
\hline 3 & 40 & 40 & 20 & 0 & 100 \\
\hline $\mathbf{v}^{\prime}$ & 20 & 40 & 30 & & \\
\hline$\overline{\mathbf{x}^{\prime}}$ & 100 & 100 & 100 & & \\
\hline
\end{tabular}

